УДК 621.315 .592

\title{
Особенности гальваномагнитных явлений в сильных магнитных полях в бикристаллах кручения 3D топологического изолятора $\mathrm{Bi}_{1-x} \mathrm{Sb}_{x}$ $(\mathbf{0 . 0 7} \leq \boldsymbol{x} \leq \mathbf{0 . 2})$
}

(C) Ф.М. Мунтяну ${ }^{1,2}$, Е.И. Георгицэ ${ }^{3}$, A. Gilewski², В. Кистол ${ }^{4}$, В. Бежан ${ }^{3}$, В. Мунтеану 1

${ }^{1}$ Институт электронной техники и нанотехнологий Академии наук Молдовы, MD-2028 Кишинев, Молдова

${ }^{2}$ Международная лаборатория сильных магнитных полей и низких температур,

53-421 Вроцлав, Польша

${ }^{3}$ Технический университет Молдовы,

MD-2004 Кишинев, Молдова

${ }^{4}$ Тираспольский государственный университет,

MD-2069 Кишинев, Молдова

E-mail: muntean_teodor@yahoo.com

(Получена 13 июля 2016 г. Принята к печати 10 августа 2016 г.)

Исследованы гальваномагнитные явления в бикристаллах кручения сплавов $\mathrm{Bi}_{1-x} \mathrm{Sb}_{x}(0.07 \leq x \leq 0.2)$ при низких температурах и в магнитных полях до 40 Тл. Установлено, что при малых углах разориентации кристаллитов переход полупроводник-полуметалл индуцируется в центральном (толщина $\sim 60$ нм) и двух смежных слоях (толщина $\sim 20$ нм каждый) интерфейса при разных значениях ультраквантового магнитного поля. В бикристаллах с большими углами разориентации в сильных магнитных полях наблюдались квантовые осцилляции магнитосопротивления и эффекта Холла, которые свидетельствуют о том, что плотность электронных состояний выше, а носители заряда тяжелее в смежных слоях интерфейсах, чем в кристаллитах. Наши результаты показывают также, что в бикристаллах кручения существуют области разной плотности квантовых электронных состояний, зависящие от угла разориентации кристаллитов и напряженности магнитного поля.

DOI: 10.21883/FTP.2017.04.44331.8371

\section{1. Введение}

Узкозонные полупроводниковые сплавы $\mathrm{Bi}_{1-x} \mathrm{Sb}_{x}$ $(0.07<x<0.15)$ являются [1-4] первыми теоретически предсказанными и экспериментально обнаруженными трехмерными (3D) топологическими изоляторами (ТИ). Эти сплавы характеризуются инвертированным (относительно Вi) упорядочением энергетических экстремумов вблизи точек $L$ зоны Бриллюэна, непрямой энергетической щелью между минимумами $L$-электронов и максимумом $T$-дырок, асимметричными поверхностными состояниями Дирака [5] и спин-поляризованными состояниями, связывающими валентную зону и зону проводимости в нетривиальном топологическом виде [6].

Известно, что гальваномагнитные явления весьма привлекательны для изучения характеристик носителей заряда в ультраквантовых магнитных полях для индуцирования электронных фазовых переходов типа полупроводник-полуметалл, полуметаллполупроводник [7-9] и т. п.

В данной работе исследуются бикристаллы сплавов $\mathrm{Bi}_{1-x} \mathrm{Sb}_{x}(0.07<x<0.15)$ в очень сильных магнитных полях для выявления особенностей взаимодействия носителей заряда в различных по размерности системах, включая границу квазидвумерного 2D сверхпроводника и $3 \mathrm{D}$ ТИ [10].

\section{2. Методика эксперимента}

Бикристаллы кручения получены методом зонной перекристаллизации с использованием технологии двойной затравки. Образцы для измерения были изготовлены в виде прямоугольных брусков $(1 \times 2 \times 4 \mathrm{Mм})$, где соотношение объема интерфейса к общему объему бикристалла $\sim 10^{-4}$. Состав образцов контролировался методами сканирующей электронной микроскопии (СЭМ) и методами оптической эмиссионной спектрометрии с использованием спектрометра Jobin-Yvon JY-38-S. Были исследованы две группы бикристаллов кручения (см. рис. 1): с малым углом разориентации кристаллитов $\left(\mathrm{SDA}, \theta_{1}<9^{\circ}\right)$ и с большим углом разориентации кристаллитов $\left(\mathrm{LDA}, \theta_{1}>12^{\circ}\right)$. Ширина интерфейса $($ СИ $) \sim 100$ нм оценена с помощью СЭМ и по значениям магнитного поля, при которых начинают проявляться квантовые осцилляции.

В то время как кристаллиты бикристаллов были 3D ТИ, интерфейсы бикристаллов $\mathrm{Bi}_{1-x} \mathrm{Sb}_{x}$ $(0.07<x<0.15)$ при $T_{\text {onset }} \leq 35 \mathrm{~K}$ проявляли один или два сверхпроводящих перехода $[11,12]$ (см. рис. 2). При одном резистивном переходе, характерном для LDA образцов, критическая температура $T_{\mathrm{c}}$ была $\sim 3.7-4.6 \mathrm{~K}$ (для различных бикристаллов), а петли гистерезиса были как у слабых ферромагнетиков (см. вставку 

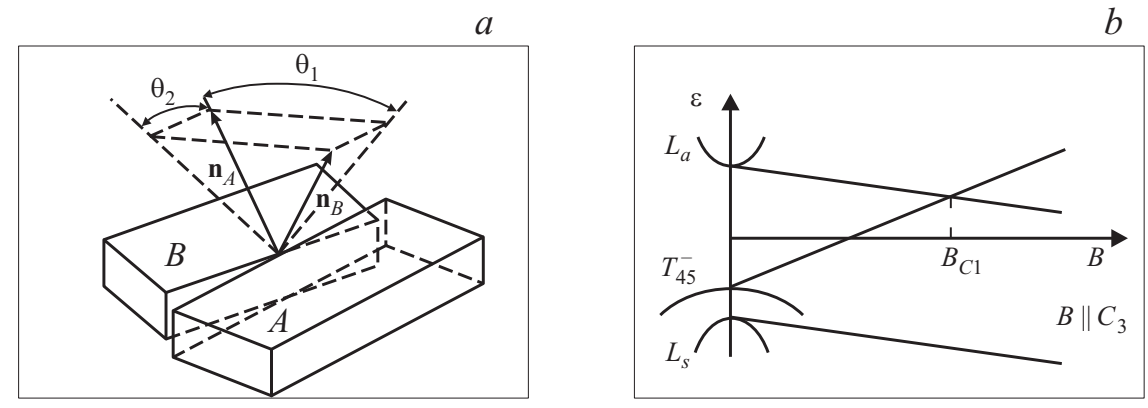

Рис. 1. $a)$ - схематическая диаграмма бикристалла. $\theta_{1}-$ угол разориентации кристаллитов, $\theta_{2}-$ угол разворота в плоскости. $b)$ - энергетическая диаграмма перестройки спектра в сильных магнитных полях (УКП) в сплавах $\mathrm{Bi}_{1-x} \mathrm{Sb}_{x}(0.07<x<0.15)$. $B_{c 1}$ - критическое магнитное поле перехода полупроводник-полуметалл.
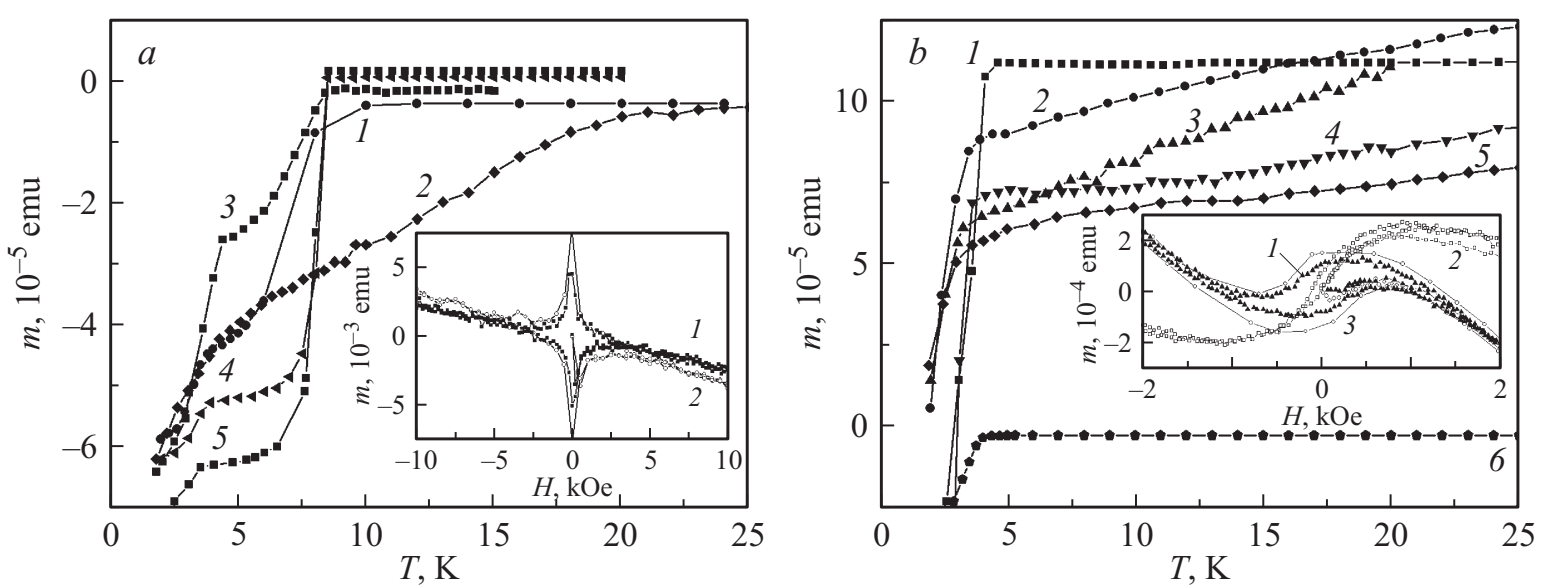

Рис. 2. Температурные зависимости магнитного момента и петли гистерезиса в бикристаллах с двумя (a) и одним (b) сверхпроводящими переходами. а) $1-\mathrm{Bi}_{0.93} \mathrm{Sb}_{0.07} \mathrm{Sn}_{0.01}, \theta_{1}=4.6^{\circ}, \theta_{2}=1^{\circ} ; 2-\mathrm{Bi}, \theta=5^{\circ}$, шкала для $m(T) 1: 4 ; 3-\mathrm{Bi}_{0.85} \mathrm{Sb}_{0.15}$, $\theta_{1}=4^{\circ}, \theta_{2}=1^{\circ} ; 4-\mathrm{Bi}_{0.93} \mathrm{Sb}_{0.07} \mathrm{Sn}, \theta_{1}=4^{\circ}, \theta_{2}=1^{\circ}$, шкала для $m(T) 1: 2 ; 5-\mathrm{Bi}_{0.94} \mathrm{Sb}_{0.06} \mathrm{Te}, \theta_{1}=9^{\circ}, \theta_{2}=2^{\circ}$, шкала для $m(T)$ $1: 2$. На вставке: $\left.\mathrm{Bi}_{0.93} \mathrm{Sb}_{0.07} \mathrm{Sn}_{0.01}, \theta_{1}=4.6^{\circ}, 1-2 \mathrm{~K}, 2-5 \mathrm{~K} ; b\right) 1-\mathrm{Bi}_{0.93} \mathrm{Sb}_{0.07} \mathrm{Sn}_{0.005}, \theta_{1}=75^{\circ}, \theta_{2}=4^{\circ}$, шкала для $m(T)$ $1: 4 ; 2-\mathrm{Bi}_{0.93} \mathrm{Sb}_{0.07} \mathrm{Sn}_{0.005}, \theta_{1}=68^{\circ}, \theta_{2}=2^{\circ}$, шкала для $m(T) 1: 10 ; 3-\mathrm{Bi}_{0.85} \mathrm{Sb}_{0.15} \mathrm{Te}_{0.001}, \theta_{1}=19^{\circ}, \theta_{2}=3^{\circ}$, шкала для $m(T)$ $20: 1 ; 4-\mathrm{Bi}_{0.94} \mathrm{Sb}_{0.06} \mathrm{Te}_{0.01}, \theta_{1}=69^{\circ}, \theta_{2}=2^{\circ} ; 5-\mathrm{Bi}_{0.82} \mathrm{Sb}_{0.18} \mathrm{Sn}_{0.005}, \theta_{1}=18^{\circ}, \theta_{2}=5^{\circ}$, шкала для $m(T) 300: 1 ; 6-\mathrm{Bi}_{0.85} \mathrm{Sb}_{0.15}$, $\theta_{1}=120^{\circ}, \theta_{2}=5^{\circ}$. На вставке: $\mathrm{Bi}_{0.85} \mathrm{Sb}_{0.15}, \theta_{1}=12^{\circ}, \theta_{2}=5^{\circ}, 1-10 \mathrm{~K}, 2-300 \mathrm{~K}, 3-1.8 \mathrm{~K}$.

на рис. 2,b). С другой стороны, для большинства SDA бикристаллов с двумя переходами $\left(T_{\mathrm{c} 1} \sim 8.3-21 \mathrm{~K}\right.$, $\left.T_{\mathrm{c} 2} \sim 3.7-4.6 \mathrm{~K}\right)$ петли гистерезиса были типичными для сверхпроводников второго рода (см. вставку на рис. 2,a).

Магнитосопротивление и эффект Холла бикристаллов изучались в магнитном поле, ориентированном вдоль плоскости СИ, т.е. вблизи осей $C_{3}$ кристаллитов. Контактные электроды из медных проволок фиксировались при помощи электроискровой сварки. Квантовые осцилляционные эффекты позволили установить, что СИ состоит из центральной части толщиной $\sim 60$ нм и двух смежных слоев толщиной $\sim 20$ нм, расположенных по обе стороны от центральной части [13].

Измерения проведены в стационарных (до 18Тл) и импульсных (до 40 Тл) магнитных полях в Международной лаборатории сильных магнитных полей и низких температур во Вроцлаве, Польша.

\section{3. Результаты исследования и их обсуждение}

Известно, что ультраквантовый предел (УКП) электронной системы реализуется в магнитных полях, когда носители заряда заселяют лишь нижайший $(j=0)$ уровень/подуровень Ландау. В УКП происходит смещение края зон $\Delta \varepsilon$ (см. рис. $1, b$ ) в зависимости от соотношения спинового $\Delta \varepsilon_{s}$ и орбитального $\Delta \varepsilon_{0}$ расщеплений уровней Ландау: $\Delta \varepsilon=0.5 h \omega\left(1-\Delta \varepsilon_{s} / \Delta \varepsilon_{0}\right)$, где $h-$ постоянная Планка, $\omega$ - циклотронная частота.

На рис. $3, a, b$ показана зависимость магнитосопротивления $\Delta \rho / \rho$ от поля в монокристаллах и SDA бикристаллах кручения сплавов $\mathrm{Bi}_{1-x} \mathrm{Sb}_{x}(0.07<x<0.15)$. В стационарных магнитных полях, ориентированных вдоль плоскости СИ, при $B \leq 2$ Тл (см. рис. 3, $a$, кривая 3 ) проявлялись регулярные шубниковские осцилляции от монокристаллов и кристаллитов бикристаллов. В более 

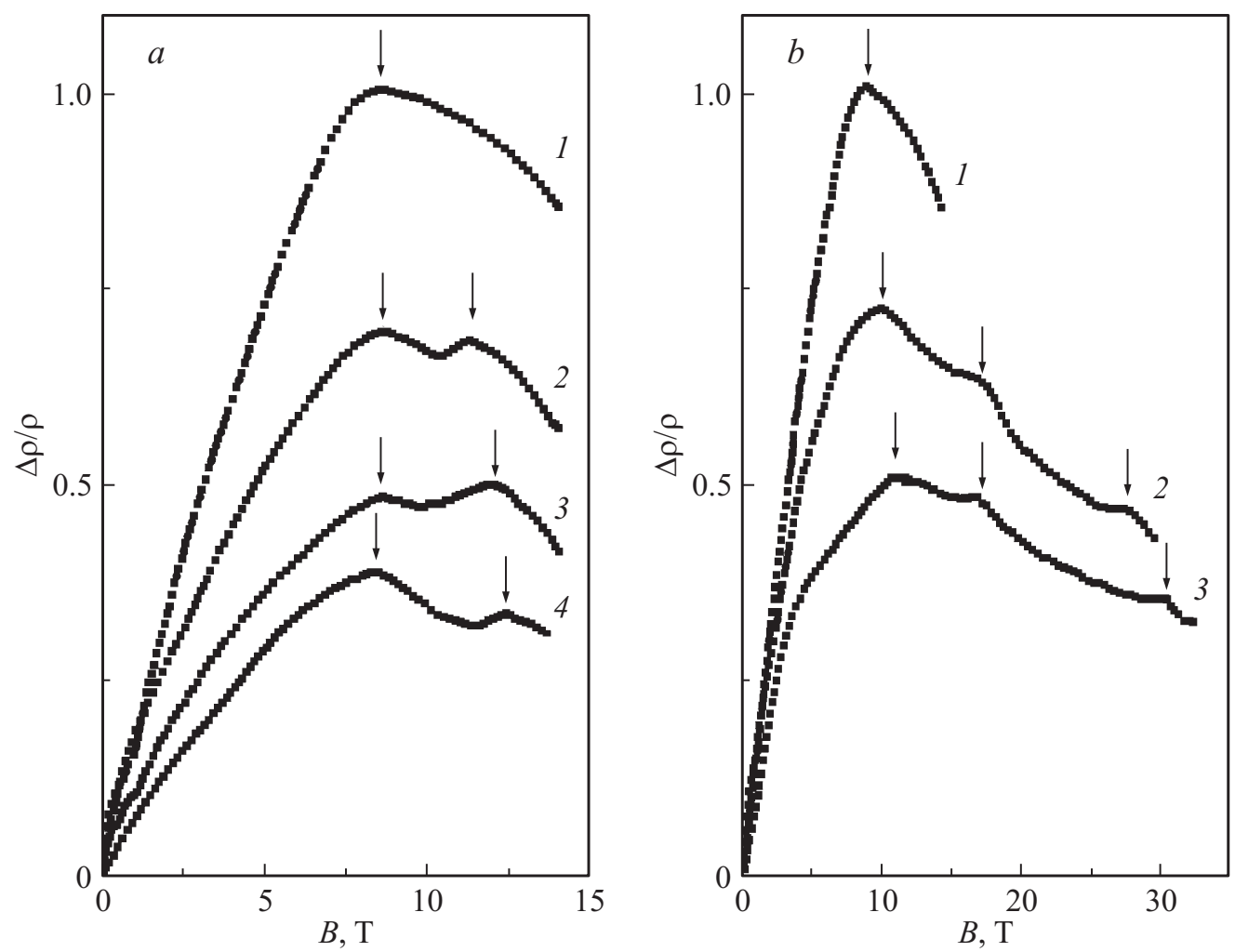

Рис. 3. Магнитосопротивление $\Delta \rho / \rho$ в квантующих магнитных полях в монокристалле и SDA бикристаллах кручения сплавов $\mathrm{Bi}_{1-x} \mathrm{Sb}_{x}(x=0.08$ и 0.09$)$ при $\left.T=4.2 \mathrm{~K} . a\right)$ стационарные магнитные поля: 1 - монокристалл, $x=0.08 ; 2,3$ и $4-$ бикристаллы с $\left.x=0.08 ; 2-\theta_{1}=4^{\circ}, \theta_{2}=2^{\circ} ; 3-\theta_{1}=1^{\circ}, \theta_{2}=4^{\circ} ; 4-\theta_{1}=9^{\circ}, \theta_{2}=2^{\circ} . b\right)$ импульсные магнитные поля: $1-$ монокристалл, $x=0.08 ; 2$ и $3-$ бикристаллы: $2-x=0.08, \theta_{1}=4^{\circ}, \theta_{2}=2^{\circ} ; 3-x=0.09, \theta_{1}=12^{\circ}, \theta_{2}=2^{\circ}$. Стрелки указывают на значения поля, где проявляются переходы полупроводник-полуметалл.

сильных полях носители заряда находились в УКП и при $B \sim 8.5$ Тл наблюдался максимум $\Delta \rho / \rho$, связанный [7-9] с переходом полупроводник-полуметалл (см. рис. $3, a)$ как в монокристалле (кривая 1), так и в бикристаллах (кривые 2, 3 и 4). Заметим, что в бикристаллах первый максимум $\Delta \rho / \rho$ заметен в том же поле, что и в монокристалле, что свидетельствует о индуцировании магнитным полем электронного фазового перехода типа полупроводник-полуметалл в кристаллитах.

В полях $B>11$ Тл в SDA бикристаллах обнаружены два других дополнительных максимума $\Delta \rho / \rho$. Второй и третий максимумы наблюдаются при разных значениях магнитного поля, они зависят от величины углов разориентации кристаллитов $\theta_{1}$ и $\theta_{2}$ и связаны с переходом полупроводник-полуметалл в смежных и центральном слоях интерфейсов бикристаллов.

Обнаружение этих максимумов при различных значениях магнитного поля показывает, что циклотронная масса соответствующих носителей заряда и отношение спинового и орбитального расщепления уровней Ландау $\Delta \varepsilon_{s} / \Delta \varepsilon_{0}$ в кристаллитах и в СИ SDA бикристаллов значительно отличаются. Это подразумевает увеличение спин-орбитального взаимодействия и существование бесщелевых электронных состояний на поверхности бикристаллов.
Какие-либо особенности $\Delta \rho / \rho$, обусловленные электронными фазовыми переходами типа полупроводник-полуметалл в LDA бикристаллах $\mathrm{Bi}_{1-x} \mathrm{Sb}_{x}$ $(0.07<x<0.15)$, не обнаружены. С другой стороны, отчетливо наблюдались (см. рис. 4) квантовые осцилляции магнитосопротивления $\rho_{i i}(B)$ и продольного эффекта Холла $\rho_{i j}\left(B_{i}\right)$ от СИ этих бикристаллов.

Спектры квантовых осцилляций LDA бикристаллов $\mathrm{Bi}_{1-x} \mathrm{Sb}_{x}$ были проанализированы графическим методом на основе зависимости положения $B_{n}^{-1}$ пиков осцилляций от номера уровня Ландау $n$.

Соотношение между $B_{n}$ и $n$ описывается формулой: $1 / B_{n}=n e /\left(h N_{s}\right)$, где $e-$ элементарный заряд, $h-$ постоянная Планка, а $N_{s}$ - поверхностная плотность состояний. Зависимости $B_{n}$-n, полученные для трех LDA бикристаллов, показаны на вставке рис. 4. Были обнаружены две частоты колебаний, связанные с поверхностью Ферми центрального и смежных слоев СИ LDA бикристаллитов. Результаты показывают, что в СИ электронные состояния относятся к типу Шредингера, так как для них $n$ принимает целые значения и фаза Берри равна нулю. Заметим, что в этих бикристаллах, в отличие от бикристаллов $\mathrm{Bi}$, гармоники, связанные с очень большими площадями поперечного сечения поверхности Ферми и с аномалиями продольного эф- 


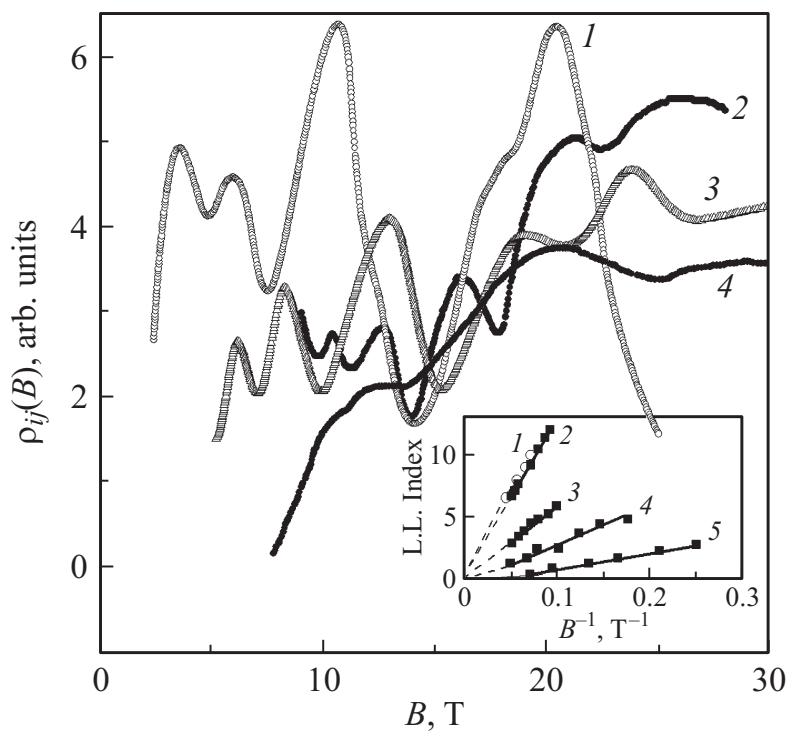

Рис. 4. Квантовые осцилляции магнитосопротивления $\rho_{i i}(B)$ и продольного эффекта Холла $\rho_{i j}\left(B_{i}\right)$ в LDA бикристаллах $\mathrm{Bi}_{1-x} \mathrm{Sb}_{x}(x=0.08,0.12,0.15)$ и $\mathrm{Bi}_{0.93} \mathrm{Sb}_{0.07}$ Те при $4.2 \mathrm{~K}$ : $1-\rho_{i i}(B), x=0.08, \theta_{1}=15^{\circ}, \theta_{2}=3^{\circ} ; 2-\rho_{i j}\left(B_{i}\right), x=0.12$, $\theta_{1}=12^{\circ}, \theta_{2}=2^{\circ} ; 3-\rho_{i i}(B), \mathrm{Bi}_{0.93} \mathrm{Sb}_{0.07} \mathrm{Te}, \theta_{1}=19^{\circ}, \theta_{2}=2^{\circ}$; $4-\rho_{i i}(B), x=0.15, \theta_{1}=15^{\circ}, \theta_{2}=3^{\circ}$. На вставке: зависимости номеров уровней Ландау $n$ от индукции магнитного поля $B_{n}^{-1}: 2$ и 5 получены для гармоники 1,1 и 3 - для гармоники 2 и 4 - для гармоники 3 .

фекта Холла (квазиплато, смещение положения пиков осцилляций и т.п.), не наблюдались [14].

Установлено, что циклотронные массы носителей заряда в слоях СИ, оцениваемые по температурным зависимостям амплитуды квантовых осцилляций, в несколько раз превышают значения в кристаллитах [15]. Например, в магнитном поле, ориентированном вдоль плоскости СИ в бикристалле $\mathrm{Bi}_{0.93} \mathrm{Sb}_{0.07} \mathrm{Te}$, циклотронная масса носителей $m_{c} / m_{e}$ (где $m_{e}-$ масса свободного электрона) в кристаллитах, смежных и центральном слоях СИ равняется $0.05,0.25$ и 0.5 соответственно. Квантовые осцилляции в LDA бикристаллах $\mathrm{Bi}_{1-x} \mathrm{Sb}_{x}$ имеют аналогичную структуру осцилляционного спектра как и в бикристаллах $\mathrm{Bi}$, хотя $\mathrm{Bi}$ - полуметалл, а сплавы $\mathrm{Bi}_{1-x} \mathrm{Sb}_{x}(0.07<x<0.15)$ - узкозонные полупроводники. Этот факт является еще одним аргументом в пользу квантового происхождения наблюдаемых эффектов для слоев СИ, свойства которых различны и независимы от характеристик основного материала. Плотность состояний $N_{s}$ в слоях СИ LDA бикристаллов $\mathrm{Bi}_{1-x} \mathrm{Sb}_{x}$ была оценена из квантовых осцилляций по методике [16]. Получены значения $N_{s} \sim(0.2-0.3) 10^{19}$ и $(1.5-2.5) \cdot 10^{19} \mathrm{M}^{-2}$ для смежных и центральных слоев соответственно, что на несколько порядков выше, чем $N_{s}$ схожих по толщине пленок сплавов $\mathrm{Bi}_{1-x} \mathrm{Sb}_{x}[17]$.

Bce эти результаты показывают, что носители заряда в слоях СИ LDA бикристаллов $\mathrm{Bi}_{1-x} \mathrm{Sb}_{x}$ $(0.07<x<0.15)$ сильно вырождены, при этом в ак- туальных энергетических зонах есть много состояний, локализующих более тяжелые носители заряда, чем в кристаллитах.

\section{4. Заключение}

Определены квантовые характерные особенности гальваномагнитных эффектов бикристаллов 3D ТИ $\mathrm{Bi}_{1-x} \mathrm{Sb}_{x}(0.07<x<0.15)$ в сильных магнитных полях. Обнаружено, что в SDA бикристаллах переход полупроводник-полуметалл индуцируется магнитным полем, сначала в кристаллитах при $\sim 8.5$ Тл, а затем в полях больше 11 Тл, в центральных и смежных слоях интерфейса. B LDA бикристаллах переход полупроводникполуметалл не был детектирован. Однако в этих бикристаллах выявлены особые квантовые осцилляции гальваномагнитных эффектов, связанные с составляющими слоями интерфейса, характеризующими более высокую плотность состояний и значительно более тяжелые носители заряда, чем в кристаллитах. Судя по этим данным, бикристаллы 3D ТИ $\mathrm{Bi}_{1-x} \mathrm{Sb}_{x}(0.07<x<0.15)$ могут быть полезны для изучения ряда важных проблем современной физики, в том числе проблем сверхпроводящих систем с узловыми точками Дирака и Маджорана (Majorana) моды [18-21], а также вопросов связанных с квантовой спин Холл фазой [6] и др.

\section{Список литературы}

[1] L. Fu, C.L. Kane. Phys. Rev. B, 76, 045302 (2007).

[2] B.A. Bernevig, T. Hughes, S.C. Zhang. Science, 314, 1757 (2006).

[3] J.E. Moore. Nature, 464, 194 (2010); Nature Physics, 11, 897(2015).

[4] L. Fu, C.L. Kane, E.J. Mele. Phys. Rev. Lett., 98, 106803 (2007).

[5] J.C.Y. Teo, L. Fu, C.L. Kane. Phys. Rev. B, 78, 045426 (2008).

[6] D. Hsieh, D. Qian, L. Wray, Y. Xia, Y. Hor, R.J. Cava, M.Z. Hasan. Nature, 452, 970 (2008).

[7] N.B. Brandt, Y.G. Ponomarev, S.M. Chudinov. J. Low Temp. Phys., 8, 389 (1972).

[8] E.W. Fenton. Phys. Rev., 170, 816 (1968).

[9] N.B. Brandt, S.M. Chudinov. J. Low Temp. Phys., 8, 339 (1972).

[10] X.L. Qi, S.C. Zhang. Rev. Mod. Phys., 83, 1057 (2011).

[11] F.M. Muntyanu, A. Gilewski, K. Nenkov, A. Zaleski, V. Chistol. Sol. St. Commun., 147, 183 (2008).

[12] F.M. Muntyanu, A. Gilewski, K. Nenkov, K. Rogacki, A. Zaleski, G. Fuks, V. Chistol. Phys. Lett. A, 378, 1213 (2014).

[13] F.M. Muntyanu, A. Gilewski, K. Nenkov, A. Zaleski, V. Chistol. Phys. Rev. B, 76, 014532 (2007).

[14] F.M. Muntyanu, A. Gilewski, K. Nenkov, A. Zaleski, T. Palewski, V. Chistol. Sol. St. Commun., 158, 9 (2013).

[15] F.M. Muntyanu, Y.A. Dubkovetskii, A. Gilevski. Phys. Sol. St., 46, 1763 (2004) [Sov. Phys. Solid State, 46, 1822 (2004)].

[16] J. Hajdu, G. Landwehr. Strong and Ultrastrong Magnetic Fields and Their Applications, ed. by F. Herlach (Springer Verlag, Berlin, 1985). 
[17] J.A. van Hulst, H.M. Jaeger, S. Radelaar. Phys. Rev. B, 52, 5953 (1995).

[[18] Z. Zhu, Y. Cheng, U. Schwingenschlögl. Sci. Rept., 4, 4025 (2014).

[19] F. Wilczek. New J. Phys., 16, 082003 (2014).

[20] F. Wilczek. Nature, 486, 195 (2012).

[21] S. Sasaki, M. Kriener, K. Segawa, K. Yada, Y. Tanaka, M. Sato, Y. Ando. Phys. Rev. Lett., 107, 217001 (2011).

Редактор А.Н. Смирнов

\section{Peculiarities of galvanomagnetic effects in} high magnetic fields in twisting bicrystals of 3D topological insulator

$\mathrm{Bi}_{1-x} \mathbf{S b}_{x}(\mathbf{0 . 0 7} \leq \boldsymbol{x} \leq \mathbf{0 . 2})$

F.M. Muntyanu 1,2, E.I. Gheorghitsa ${ }^{3}$, A. Gilewski $^{2}$, V. Chistol ${ }^{4}$, V. Bejan ${ }^{3}$, V. Munteanu ${ }^{1}$

${ }^{1}$ Institute of Electronic Engineering and Industrial Technologies,

Academy of Sciences of Moldova, MD-2028 Chisinau, Moldova

${ }^{2}$ International Laboratory of High Magnetic Fields and Low Temperatures, 53-421 Wroclaw, Poland

3 Tiraspol State University, MD-2069 Chisinau, Moldova

4 Technical University of Moldova, MD-2004 Chisinau, Moldova

Abstract Galvanomagnetic effects in twisting bicrystals of $\mathrm{Bi}_{1-x} \mathrm{Sb}_{x}(0.07 \leq x \leq 0.2)$ at low temperatures and in magnetic fields up to $40 \mathrm{~T}$ were studied. It has been found that for bicrystals with small crystallite disorientation angle, the semiconductorsemimetal transition is induced in crystallites, in the central (thick $\sim 60 \mathrm{~nm}$ ) and in two adjacent (thick $\sim 20 \mathrm{~nm}$ each) layers of crystallite interfaces at different values of ultra-quantum magnetic field. For bicrystals with large crystallite disorientation angle the quantum oscillations of the magnetoresistance and Hall effect were observed, showing that the density of states is higher and the charge carriers are heavier in crystallite interfaces than in crystallites. Our results show also that in twisting bicrystals there are areas of different quantum electronic states depending of the crystallite disorientation angle and magnetic field. 